\title{
Sigma-2 receptor ligands potentiate conventional chemotherapies and improve survival in models of pancreatic adenocarcinoma Hiroyuki Kashiwagi ${ }^{1}$, Jonathan E McDunn ${ }^{2}$, Peter O Simon Jr${ }^{1}$, Peter S Goedegebuure1,3, Suwanna Vangveravong4, Katherine Chang2, Richard S Hotchkiss², Robert H Mach ${ }^{4}$ and William G Hawkins*1,3
}

\begin{abstract}
Address: ${ }^{1}$ Department of Surgery, Washington University School of Medicine, 660 S. Euclid Avenue, Campus Box 8109, St. Louis, MO 63110, USA ${ }^{2}$ Department of Anesthesiology, Washington University School of Medicine, 660 S. Euclid Avenue, St. Louis, MO 63110, USA, ${ }^{3}$ Alvin J. Siteman Cancer Center, Washington University School of Medicine, 660 S. Euclid Avenue, Campus Box 8109, St. Louis, MO 63110, USA and ${ }^{4}$ Department of Radiology, Washington University School of Medicine, 660 S. Euclid Avenue, St. Louis, MO 63110, USA

Email: Hiroyuki Kashiwagi - hiroyukiwagi1967@yahoo.co.jp; Jonathan E McDunn - mcdunnj@morpheus.wustl.edu; Peter O Simon - simonpo@wudosis.wustl.edu; Peter S Goedegebuure - goedegep@wudosis.wustl.edu; Suwanna Vangveravong - vangveravongs@mir.wustl.edu; Katherine Chang - changk@morpheus.wustl.edu;

Richard S Hotchkiss - hotchkir@anest.wustl.edu; Robert H Mach - rhmach@mir.wustl.edu; William G Hawkins* - hawkinsw@wustl.edu

* Corresponding author
\end{abstract}

Published: 26 March 2009

Journal of Translational Medicine 2009, 7:24 doi:10.1 I86/1479-5876-7-24

Received: 14 November 2008

Accepted: 26 March 2009

This article is available from: http://www.translational-medicine.com/content/7///24

(C) 2009 Kashiwagi et al; licensee BioMed Central Ltd.

This is an Open Access article distributed under the terms of the Creative Commons Attribution License (http://creativecommons.org/licenses/by/2.0), which permits unrestricted use, distribution, and reproduction in any medium, provided the original work is properly cited. 


\section{Background}

Pancreas cancer is the fourth leading cause of cancerrelated mortality in the United States [1]. The 5-year survival rate is less than 5\% [2]. This poor outcome stems from the difficulty in achieving an early diagnosis and the failure of surgery, radiation and chemotherapy. In fact, only $15 \%$ of patients are eligible for surgical resection at the time of diagnosis [3]. Even after radical pancreatectomy, most patients with pancreas cancer show local recurrence or metastasis within 1 year. The current standard chemotherapeutic, gemcitabine, demonstrates a slight improvement in survival, but these modest results are not satisfactory [4]. Novel therapeutic strategies are desperately needed.

Standard therapies for pancreatic cancer have two major limitations. First, systemic administration of chemotherapy does not selectively target the cancer and is limited by systemic toxicity. Second, local therapies such as radiation or surgery do not address the potential for distant metastases. For these reasons, a targeted strategy which directly delivers the cytotoxic molecule to the cancer is highly desirable.

There is considerable interest in stimulating apoptosis and inhibiting survival machinery as components of cancer therapy [4-6]. Many oncogenic transformations result from the inactivation or deletion of pro-apoptotic genes or the translocation of an anti-apoptotic gene downstream of highly active promoters $[5,7,8]$. The sigma- 2 receptor is a unique targeting receptor that induces tumor apoptosis for pancreas cancer. The sigma receptor was initially proposed as a subtype of opioid receptors [9]. Early receptor binding studies using benzomorphan opioids indicated at least two subtypes of sigma receptors exist: sigma-1 and sigma- 2 subtype [5]. These subtypes display different tissue distributions and distinct physiological and pharmacological profiles in both the central and peripheral nervous systems. Although natural ligands for these receptors are still unknown, recent research has demonstrated that sigma receptors are over-expressed in a variety of human and rodent tumors $[5,6,10,11]$ and that synthetic ligands to this receptor could play an important role in cancer diagnosis and therapy [12]. We have previously reported that the sigma-2 receptor is highly expressed in pancreas cancer and weakly expressed in normal pancreas [13]. In this same study, we carefully characterized the receptor-ligand binding interaction and reported the $K \mathrm{~d}$ and $B$ max values of sigma- 2 receptor ligands in models of pancreatic adenocarcinoma. Furthermore, we have demonstrated that sigma-2 receptor specific ligands induce apoptosis in a dose-dependent fashion and that this activity occurs, at least in part, via the intrinsic apoptotic pathway. Because sigma-2 receptorspecific ligands selectively induce apoptosis in pancreas cancer, these ligands may act as sensitizers to standard chemotherapies.

Since pancreatic cancer has proven to be resistant to modern, conventional therapies, we have chosen to focus our efforts and developing novel therapeutics that specifically target this cancer. In this study, we follow up on our previous characterization of sigma-2 receptor ligands by demonstrating that these novel agents augment conventional therapies for pancreas cancer and are an exciting class of compounds for potential treatment of these malignancies.

\section{Methods}

\section{Sigma receptor ligands}

Sigma-2 specific ligands SV119, SV95, and fluorescent labeled sigma-2 ligand, SW120, were synthesized and prepared as previously described [13-15]. The Sigma-1 receptor ligand, (+)-pentazocine (Sigma Chemical, St. Louis, $\mathrm{MO})$, was used as a control.

\section{Cell lines}

Murine pancreatic adenocarcinoma, Panc-02, was obtained from Bryan Clary (Duke University) and maintained in supplemented RPMI 1640 containing glutamine $(2 \mathrm{mmol} / \mathrm{L})$, pyruvate $(1 \mathrm{mmol} / \mathrm{L})$, penicillin $(100 \mathrm{IU} /$ $\mathrm{mL})$, streptomycin $(100 \mathrm{IU} / \mathrm{mL})$, and 10\% FBS. Human pancreatic adenocarcinoma cell lines (Panc-1, AsPC-1, and CFPAC-1) were obtained from ATCC (Bethesda, MD) and maintained in Dulbecco's modified eagle's medium (DMEM) containing glutamine $(2 \mathrm{mmol} / \mathrm{L})$, pyruvate (1 $\mathrm{mmol} / \mathrm{L})$, penicillin $(100 \mathrm{IU} / \mathrm{mL})$, streptomycin (100 IU/ $\mathrm{mL}$ ), and 10\% FBS. HPDE (Human Pancreas Duct Epithelium) was obtained from Dr. Ming Sound Tsao and cultured in Keratinocyte serum-free (KSF) medium (Gibco/ Invitrogen, Carlsbad, CA) with $50 \mathrm{mg} / \mathrm{ml}$ bovine pituitary extract (BPE), $5 \mathrm{ng} / \mathrm{ml}$ epidermal growth factor (EGF), and $1 \times$ antibiotic-antimycotic cocktail (Gibco/Invitrogen). All cell culture processes were carried out in a humidified atmosphere of $5 \% \mathrm{CO}_{2}$ at $37^{\circ} \mathrm{C}$. All cultures were free of Mycoplasma as assayed by the Washington University Division of Comparative Medicine. Cultures were maintained for no longer than 12 weeks after recovery from frozen stocks.

\section{Sigma-2 ligand binding in vitro}

Tumor cells were incubated with $10 \mathrm{nM}$ of SW120 (a fluorescent-labeled sigma- 2 receptor ligand) for 30 minutes. HPDE cells were used as a normal control. To demonstrate the specificity of SW120 for Sigma-2 receptor binding, $10 \mu \mathrm{M}$ of SV95 (Sigma-2 ligand) or (+)-pentazocine (sigma-1 receptor ligand) were added to cells 30 minutes prior to SW120 treatment. All lines were then washed 3 times with PBS and evaluated by flow cytometry. 


\section{Evaluation of cytotoxicity in vitro}

Tumor cells were harvested and seeded at a density of approximately $0.2 \times 10^{6}$ cells per well in 12 -well plates in $1.0 \mathrm{ml}$ culture medium. Seeded cells were split and preincubated for more than 24 hours (Panc-02) and 48 hours (CFPAC-1, AsPC-1, and Panc-1) to maintain their growth conditions. SV119 and SW120 were dissolved in DMSO, and gemcitabine and paclitaxel were dissolved in PBS. The solutions were then added to the culture medium at the concentrations indicated with final concentration of DMSO at less than $1 \%$. The extent of apoptosis was subsequently measured as previously described [13]. Briefly, staining was performed on trypsin-EDTA treated cultures fixed with 1\% paraformaldehyde and 90\% methanol. Fixed cells were resuspended in TUNEL reagent or cleaved caspase- 3 antibody and incubated overnight at room temperature (TUNEL) or $4{ }^{\circ} \mathrm{C}$ (Caspase 3 ). After incubated cells were washed, cells were resuspended in fluorescent antibody or 7-AAD buffer and incubated for 1 hour at room temperature. Cell-associated fluorescence was determined by the flow cytometry (FACScan, BD Biosciences) and analyzed with CellQuest software (BD Biosciences).

\section{In vivo assessment of apoptosis}

Female C57BL/6 mice (8-12 weeks old) were purchased from the NCI and acclimated for at least 1 week before tumor implantation. All mice were injected in the right flank with $200 \mu \mathrm{l}$ single cell suspension containing $1.0 \times$ $10^{6}$ Panc- 02 cells. Two weeks after tumor implantation, at which point the mean tumor diameter was approximately $5 \mathrm{~mm}$, mice were treated with a single intraperitoneal injection of SV119, conventional chemotherapy, or both. Twenty-four hours later, tumors were harvested and minced to $1 \mathrm{~mm}$ and digested in a RPMI buffer containing $1 \mathrm{mg} / \mathrm{ml}$ collagenase (Sigma-Aldrich, St. Louis, MO) and $0.1 \mathrm{mg} / \mathrm{ml}$ DNase (Sigma-Aldrich, St. Louis, MO) for 45 min to obtain a single-cell suspension. After filtering, erythrocyte contaminants were lysed in Ammonium Chloride (ACK) buffer, pelleted, and resuspended in PBS (pH 7.4). Single cell suspensions were fixed by 1\% paraformaldehyde by following the above procedure. Apoptosis was then assessed as described above utilizing flow cytometry.

\section{In vivo assessment of tumor growth and survival}

Female C57BL/6 mice (8-12 weeks old) were purchased from the NCI and acclimated for at least 1 week before tumor implantation. All mice were injected in the right flank with $200 \mu \mathrm{l}$ single cell suspension containing $1.0 \times$ $10^{6}$ Panc-02 cells. Treatment of tumors started 2 weeks after tumor implantation, at which point the mean tumor diameter was approximately $5 \mathrm{~mm}$. To evaluate the effect of treatment both systemically and on tumors in vivo, several treated mice were sacrificed and blood cytologic (complete blood count) and biochemical analysis (liver enzymes, bilirubin, amylase, lipase, BUN, creatinine, glucose) were performed. For the survival study, tumor bearing mice ( $\mathrm{n}=8-10$ per group) were treated with SV119 and/or chemotherapy once daily for 7 days (paclitaxel treatment model) or every other day for 14 days (gemcitabine treatment model). Mean tumor diameter was measured three times each week. All mice were euthanized when their tumor ulcerated, reached a mean diameter of $15 \mathrm{~mm}$, or 50 days after initiation of the study. All studies were performed in accordance with an animal protocol approved by the Washington University Institutional Animal Care Facility.

\section{Statistical analysis}

Error bars, unless stated otherwise, represent means plus or minus SEM of an experiment with at least three biological replicates. For statistical analysis of differences between groups, one-way ANOVA was performed. For in vivo experiments, Kaplan-Meier survival curves were plotted and differences were compared with a log-rank test. A $p$-value less than 0.05 was considered significant for all analysis.

\section{Results}

Sigma-2 ligands have a high affinity for pancreatic adenocarcinoma cell lines compared to normal cell lines We have previously reported that murine (Panc-02) and human (AsPC-1, CFPAC-1, and Panc-1) pancreatic adenocarcinoma cell lines display increased expression of the sigma-2 receptor [13]. However, we have not previously compared the binding of Sigma-2 ligands to the normal human pancreas cell line HPDE. As demonstrated in Figure 1, Panel A, there is a high affinity of Sigma-2 ligand to the human pancreatic adenocarcinoma cell line AsPC-1 compared to the immortalized normal pancreatic cell line HPDE. This binding also appeared to be specific to the Sigma-2 receptor as we were able to demonstrate competitive inhibition by pretreating with a second Sigma-2 ligand, but not a Sigma-1 receptor ligand (pentazocine, Panel B)

\section{The apoptotic effect of the sigma-2 ligand, SVI I9, is enhanced by conventional chemotherapy in vitro} In order to evaluate the potential therapeutic effect of the sigma-2 ligand, SV119, in combination with conventional chemotherapy, we treated pancreatic cancer cell lines with SV119 and the chemotherapeutic agents gemcitabine and paclitaxel. After 24 hours of treatment in the presence of SV119 and gemcitabine or paclitaxel, all cell lines demonstrated an additive increase in apoptosis as demonstrated by increases in TUNEL staining (Figure 2). Similar responses were noted in all cell lines when cleaved caspase 3 was utilized as the endpoint (data not shown). 
A. Human Pancreatic Adenocarcinoma

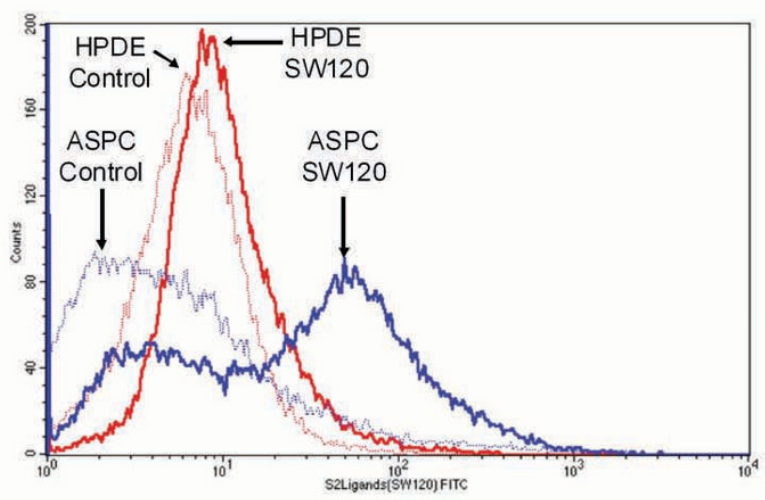

B. Murine Pancreatic Adenocarcinoma

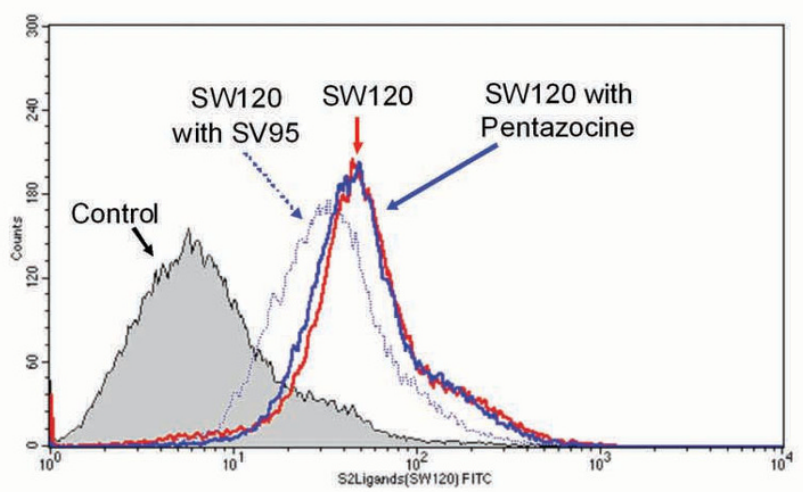

Figure I

Sigma-2 ligands have a high affinity for pancreatic adenocarcinoma cell lines compared to normal cell lines. Representative FACS analysis of human (A.) and murine (B.) pancreatic adenocarcinoma cell lines treated with the FITC-conjugated Sigma-2 ligand, SWI20. In Panel A, HPDE (immortalized pancreatic ductal epithelial cells) were used as a control. In Panel B, competitive inhibition of SWI 20 binding was demonstrated by preincubation with the Sigma-2 ligand, SW95. Pentazocine, a Sigma-I receptor ligand, was also used as a control and did not demonstrate competitive inhibition. Experiments were performed in triplicate with comparable results.

The sigma-2 ligand SVI 19 induces moderate apoptosis in both G0 and GI to G2/S phase of pancreatic cancer cells in vitro

Next, in order to further characterize this effect, we evaluated the growth phase of these pancreatic cancer cells under these conditions by co-staining for cleaved caspase3 and the proliferation maker Ki-67. As seen in Figure 3, SV119 and gemcitabine or paclitaxel induced apoptosis in cells that were both in G0 as well as in G1 to G2/S phase of the cell cycle. Mean TUNEL-positivity ranged from $16.1 \%$ to $18.6 \%$ at $10 \mu \mathrm{M}$ SV119 (Figure 3). Combining

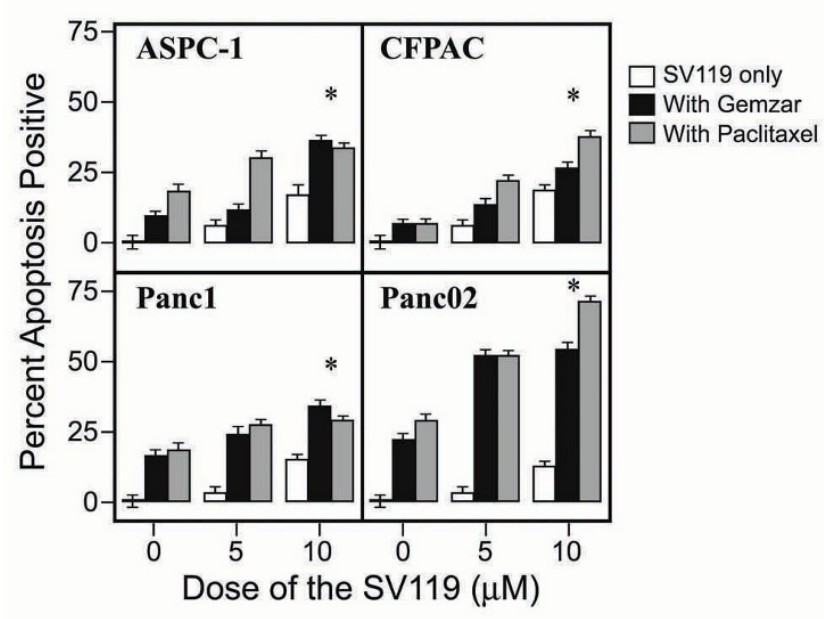

Figure 2

The apoptotic effect of the sigma-2 ligand, SVII9, is enhanced by conventional chemotherapy in vitro. Model pancreatic adenocarcinoma cell lines were treated with escalating doses of SVI I9, SVI I 9 and gemcitabine, or SVI 19 and paclitaxel. After 24 hours of treatment, percent caspase- 3 positive cells were determined by flow cytometry. Results are expressed as the mean, with bars representing standard error of the mean. Experiments were performed in triplicate with comparable results. Where indicated, $*=P<$ 0.01 for SVI 19+gemcitabine or SVI 19+paclitaxel vs. SVII9only control.

SV119 with a chemotherapy increased apoptosis. Mean TUNEL-positivity ranged from $26.5 \%$ to $70.5 \%$ in the SV119 and gemcitabine combination (50 nM) and from $26.6 \%$ to $53.8 \%$ in the SV1 19 and paclitaxel combination (50 $\mathrm{nM}$ ). As shown in the representative FACS histogram, SV119 $(10 \mu \mathrm{M})$ induced moderate apoptosis in Ki67 negative cells (G0 phase). Gemcitabine treatment shifted the cell proliferation from G0 to the active stage with moderate apoptosis (Figure 3). Paclitaxel demonstrated limited apoptosis in both G0 and active phases of the cancer cell cycle. These data suggest that SV119 may serve as a sensitizer to these conventional therapies.

The pro-apoptotic activity of the sigma-2 ligand, SVI I9, is enhanced by conventional chemotherapy in vivo without cytologic or chemical evidence of systemic toxicity

In order to determine if the pro-apoptotic effect of these agents was also conferred to tumors in vivo, an implantable murine tumor model was utilized. In this study, pancreatic tumors were implanted into the flank of C57BL/6 mice. Fourteen days after tumor implantation, a single intraperitoneal treatment on SV119, or SV119 combined with conventional chemotherapy (gemcitabine or paclitaxel) was administered. Twenty-four hours later, single cell suspensions of these tumors were generated and apoptosis was measured by FACS analysis. As shown in 

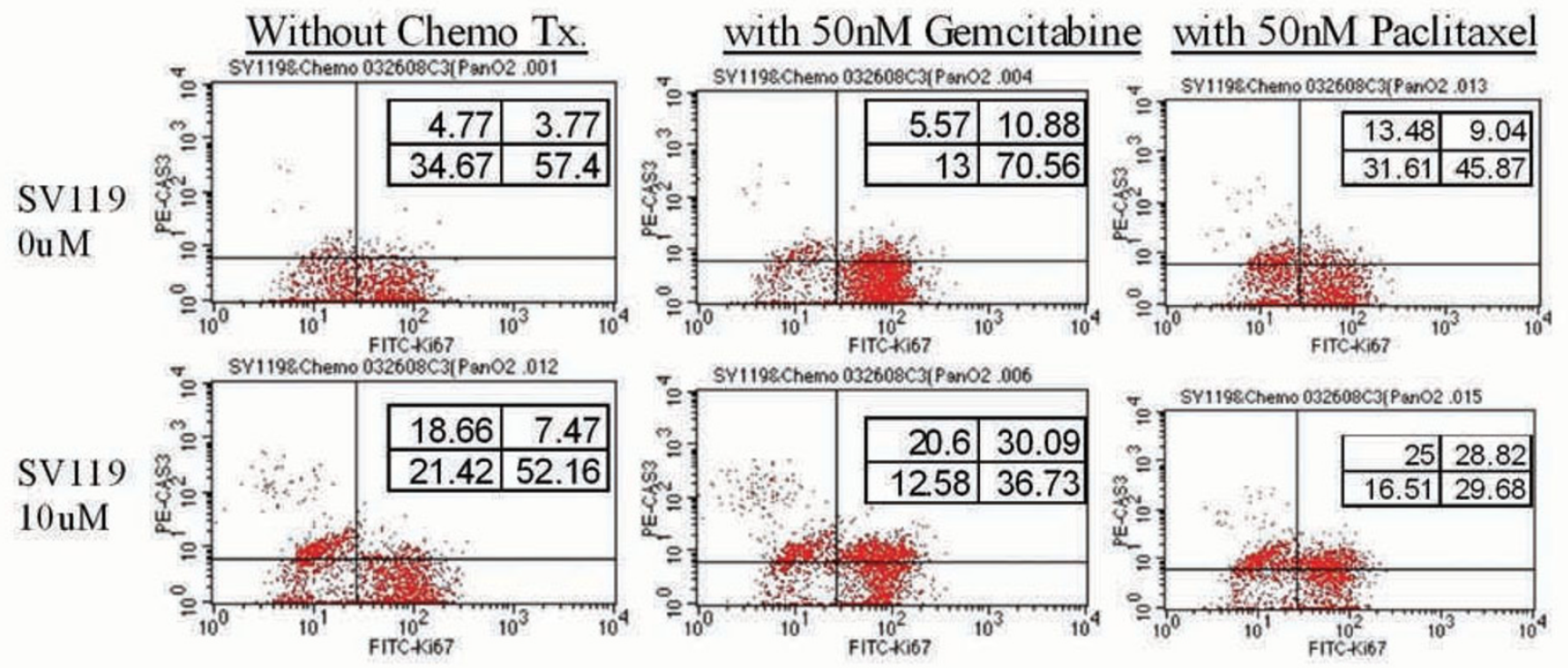

Figure 3

The sigma-2 ligand SVI 19 induces moderate apoptosis in both G0 and G I to G2/S phase of pancreatic cancer cells in vitro. The murine pancreatic adenocarcinoma cell, Panc02, was treated with SVI I 9 alone or in combination with gemcitabine or paclitaxel. After 24 hours of treatment, samples were stained for cleaved caspase-3 and Ki67. Representative histograms are shown from an experiment performed in triplicate.

Figure 4, apoptosis was markedly increased in samples that were treated with both sigma-2 ligand (SV119) and conventional chemotherapy (gemcitabine or paclitaxel). These mice appeared healthy and cytologic/biochemical laboratory analysis did not reveal major toxicity (Additional file 1) [16]. Necropsy was also performed on selected animals and no gross or histologic evidence of organ dysfunction was observed (data not shown).

\section{Treatment of mice bearing pancreatic tumor allografts with the sigma-2 receptor ligand, SVI I9, and conventional chemotherapy slows tumor growth and confers a survival advantage}

Two different treatment models of SV119 in combination with conventional chemotherapies were utilized. In the first model, weekly treatment of gemcitabine $(1.5 \mathrm{mg} /$ week) in combination with every other day treatment of SV119 was given for 2 weeks (Figure 5). In the second model, paclitaxel $(0.3 \mathrm{mg} /$ day $)$ and SV119 were used as concurrent daily treatments (Figure 6). A suboptimal dosing regimen was selected to maximize our chances of detecting a combined effect.

In vivo systemic administration of SV119-alone given as 7 daily doses or as 7 doses every other day for 14 days demonstrated a non significant tumor volume and survival advantage. Treatment with chemotherapies alone (gemcitabine or paclitaxel) also demonstrated a limited effect in both treatment models. However, in both models, the combination of SV119 with a chemotherapeutic agent significantly slowed tumor growth when compared to therapy with single agents or with untreated controls. Animals tolerated the combination therapy well, without evidence of cytologic or biochemical toxicity (data not shown).

\section{Discussion}

Pancreas cancer remains a devastating malignancy and novel therapeutic strategies are desperately needed. Cancers by definition create and develop in a stressful environment (overcrowding, hypoxia, nutrient starvation) which should promote apoptosis. Therefore most cancers including pancreas cancer develop numerous strategies which promote survival and overcome natural signals to undergo apoptosis [17]. In fact, many experts suggest that suppression of apoptosis is central to the evolution of cancer. It is also an important factor for resistance to many standard cancer treatments [12,18-21]. Standard therapies including most chemotherapeutics and radiation therapy induce cellular stress and thereby promote apoptosis. Standard therapies capitalize on the premise that cells in stressful microenvironments have increasing susceptibility to apoptogenic stimuli when subjected to additional cellular stressors such as cytotoxic therapeutics. This argument appears to be true for the common therapeutics utilized in the treatment of pancreas cancer. For example gemcitabine inhibits DNA replication, indirectly promoting apoptosis, and paclitaxel arrests the cell cycle, directly promoting apoptosis. 

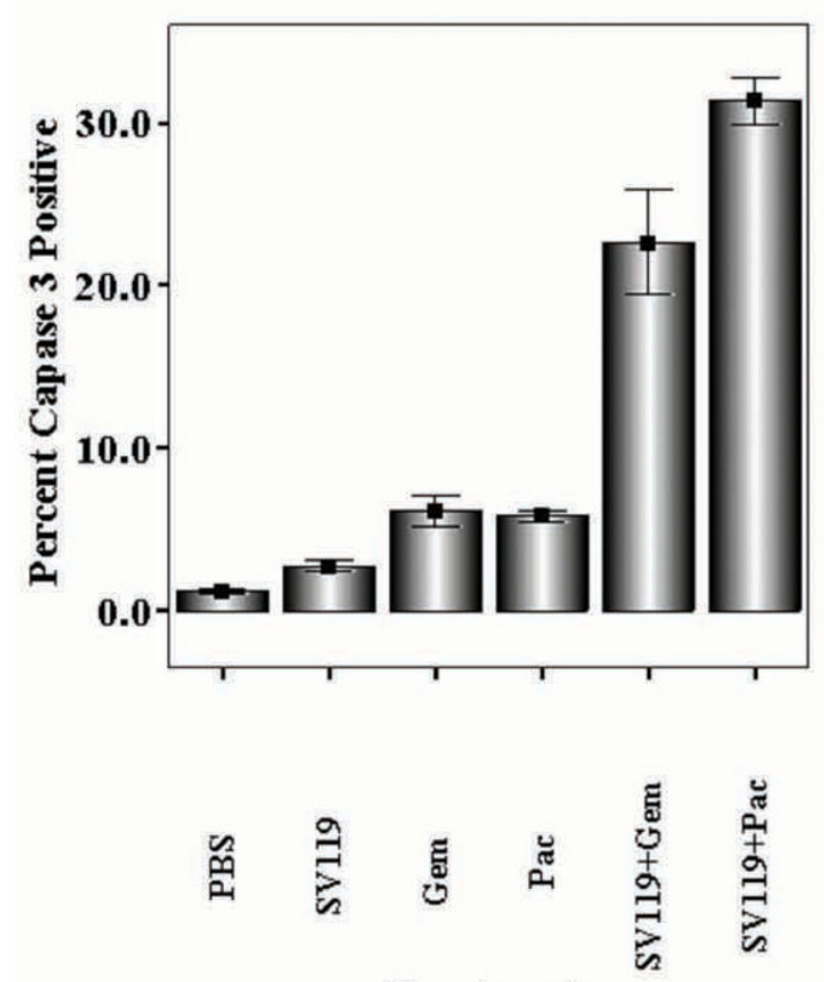

\section{Treatment}

\section{Figure 4}

The pro-apoptotic activity of the sigma-2 ligand, SVI I9, is enhanced by conventional chemotherapy in vivo. $\mathrm{C} 57 \mathrm{BL} / 6$ mice bearing implanted tumor allografts were treated with a single dose of SVII 9 and conventional chemotherapy (gemcitabine or paclitaxel). Twenty-four hours after treatment, tumors were harvested and single cell suspensions were generated. Percent active caspase- 3 was then measured in tumor cells by flow cytometry. Each experimental group represents an $n=3$. Results are expressed as the mean, with bars representing standard error of the mean.

The sigma- 2 receptor and its undiscovered endogenous ligand(s) is poorly understood. Literature regarding the role of the sigma-2 receptor in normal homeostasis is unquestionably lacking. Most of what is understood about this receptor comes from investigations in tumors. Several groups of investigators have shown that sigma-2 receptor expression is markedly increased across diverse malignancies. Recent data have suggested that this upregulation is related to cell proliferation [5]. This feature has generated interest in utilizing sigma-2 ligands as radiotracers for cancer imaging. Our group has shown that once the receptor is engaged certain ligands are rapidly internalized and distributed to membrane-encapsulated organelles [11]. This finding is consistent with the report by Ostenfeld et al that siramesine, a sigma-2 receptor selective ligand, is lysosomotrophic [15].
(A)

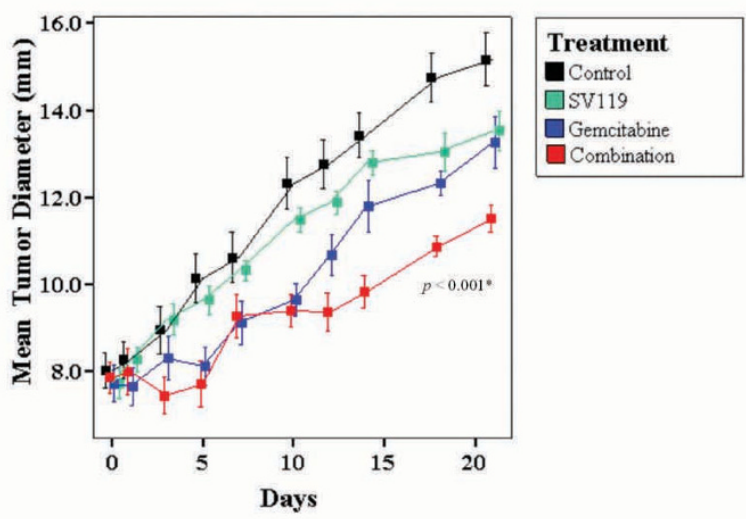

(B)

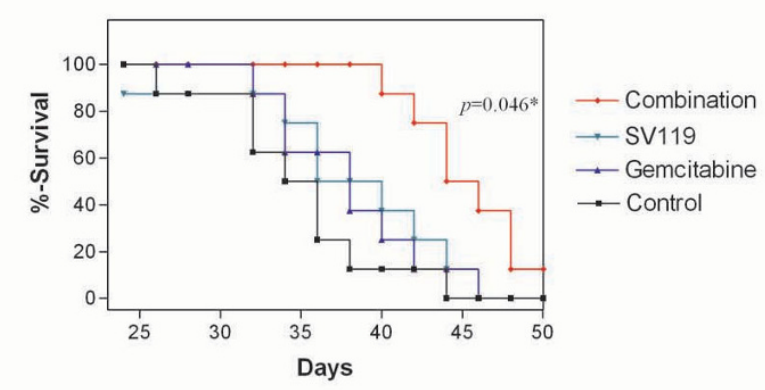

Figure 5

The sigma-2 ligand, SVI I9, combined with gemcitabine suppresses tumor growth and increases survival in model pancreatic adenocarcinoma in vivo. C57BL/6 mice bearing established tumor allografts were treated with every other day SVII 9 (I mg/mouse, i.p. for 7 days) and weekly gemcitabine ( $3 \mathrm{mg} / \mathrm{mouse}$, i.p. for two weeks). Mean tumor diameter (Panel A) and survival (Panel B) were measured. $*=$ vs. control.

We and others have recently shown that selected sigma-2 ligands are capable of inducing apoptosis in a multitude of human and murine cancer cells lines and in animal models of cancer including pancreas cancer $[12,13,15]$. The mechanisms by which this works are poorly understood but we do know that the apoptosis generated by selected sigma-2 ligands can be partially inhibited with intrinsic pathway inhibitors like caspase inhibitor [13]. While the anti-tumor effects of sigma-2 ligands alone are modest, the high receptor abundance on cancers and the high affinity of the ligands for the receptor may present a unique opportunity to utilize these ligands as chemotherapeutic sensitizers.

We hypothesized that sigma-2 ligands may selectively augment the effects of non-selective pro-apoptotic anti- 
(A)

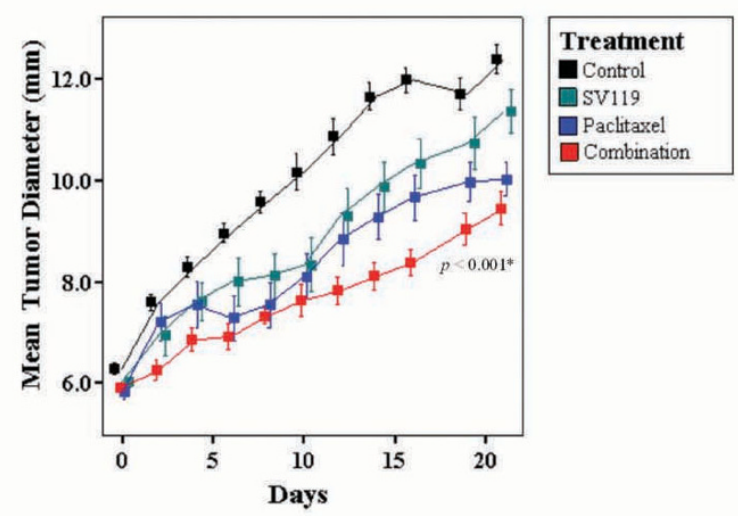

(B)

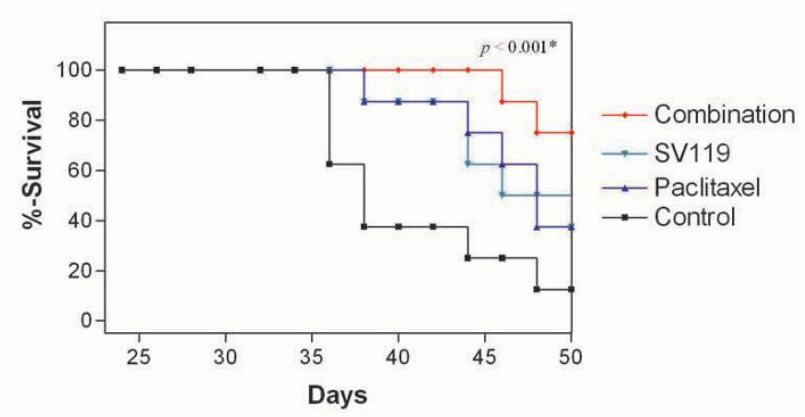

Figure 6

The sigma-2 ligand, SVI I9, combined with paclitaxel suppresses tumor growth and increases survival in model pancreatic adenocarcinoma in vivo. C57BL/6 mice bearing established tumor allografts were treated with daily SVI 19 (I mg/mouse, i.p. for 7 days) and daily paclitaxel ( $0.3 \mathrm{mg} / \mathrm{mouse}$, i.p. for 7 days). Mean tumor diameter (Panel A) and survival (Panel B) were measured. $*=$ vs. control.

cancer therapies preferentially in cancer cells. The high tumor receptor abundance may provide a novel strategy for improving on the effects of cytotoxic chemotherapies without increasing toxicity. Since sigma-2 ligands are expressed on other tissues (although at lower levels) we were concerned that such a combined strategy might result in toxicity wherever sigma-2 ligands are found. We tested whether SV119 (an apoptogenic sigma-2 ligand) and a standard chemotherapeutic would slow tumor growth, reduce toxicity, and ultimately improve survival in a murine model of established pancreas adenocarcinoma.
In our present study, both the specific ligand of the Sigma2 receptor (SV119) and the chemotherapies showed moderate apoptosis in all pancreas cancer cells in vitro. SV119 induced tumor apoptosis in both cycling cells at all phases (G1 to G2M/S) and in quiescent, G0, cells (Figure 3). Depending on the cell line assayed, SV119 in combination with the lower dose of chemotherapies showed an additive or super-additive effect in inducing tumor apoptosis (Figure 3). These results indicate that SV119 is a useful sensitizer for pancreas cancer treatment in combination with cell cycle specific chemotherapies. In addition, the combination of SV119 with standard chemotherapy may decrease the chemotherapy dose required. This is significant because it is typically the systemic toxicity of contemporary chemotherapeutics that limit their effectiveness.

In the allograft C57/BL6 model of pancreas cancer, SV119 treatment in combination with gemcitabine or paclitaxel led to tumor stability and regression in some cases when compared to single therapies. Although all tumors resumed growing shortly after treatment was stopped, tumors in mice receiving combination treatment grew more slowly than tumors in either of the single agent treatments or vehicle-injected control. This result suggests that combination therapy was not only successful in reducing tumor mass but also altered the course of tumor growth after therapy was stopped. Importantly no significant toxicities were appreciated by serum biochemistry or by necropsy and immunohistochemistry.

\section{Conclusion}

Pancreas cancer is an aggressive and rapidly metastasizing tumor and we believe that it is unlikely that a single therapeutic will result in a cure for this devastating cancer. Here, we have demonstrated that the sigma-2 receptorspecific ligand, SV-119, potentiates cell death when combined with conventional chemotherapies without appreciable toxicity in model pancreatic adenocarcinoma. It is highly critical to investigate novel strategies which might complement or enhance other proven anti-cancer regimens for the treatment of pancreas cancer. We believe that this experimental design highlights a new potential strategy for the treatment of pancreas cancer and warrants further exploration.

\section{Competing interests}

The authors declare that they have no competing interests.

\section{Authors' contributions}

HK Performed experiments, interpreted results, drafted manuscript. JEM Drafted manuscript, critical revision to manuscript, designed experiments, interpreted results. POS Drafted manuscript, critical revision to manuscript, designed experiments, interpreted results. PSG Critical 
revision to manuscript. SV Designed and conducted experiments. KC Designed and conducted experiments. RSH Critical revision to manuscript, designed experiments, interpreted results. RHM Synthesis of sigma-2 ligands, critical revision to manuscript. WGH Designed experiments, interpreted results, final draft of manuscript. All authors have read and approved the final manuscript.

\section{Additional material}

\section{Additional file 1}

Table S1 - Serum toxicology and cytology of mice treated with the sigma-2 ligand, SV119, and conventional chemotherapy. Peripheral blood was drawn from tumor-bearing mice 24 hours after treatment with a single dose of SV119 and conventional chemotherapy (gemcitabine or paclitaxel). Cytologic and serum chemistry evaluations were performed by the animal care facility at Washington University. Data is expressed as mean $+/$ - standard error of the mean. Each experimental group represents an $n=2$.

Click here for file

[http://www.biomedcentral.com/content/supplementary/14795876-7-24-S1.doc]

\section{Acknowledgements}

This study was supported by grants from the American Association for Cancer Research (07-40-25-KASH, H. Kashiwagi), the National Institutes of Health (T32 CA0962I, P.O. Simon), GM44I I 8, GM55I 94 (R.S. Hotchkiss), the American Cancer Society (MRSG-08-0I9-0ICDD, W.G. Hawkins), and the Barnes-Jewish Hospital Foundation (W.G. Hawkins). Histopathology specimens were prepared at the Washington University Digestive Diseases Research Core Center (DDRCC) which is supported by the National Institutes of Health (P30 DK052574). This work was presented in part at the Annual Meeting of the Society for Surgical Oncology Cancer Forum, Chicago, 2008. The authors would like to thank Morgan Younkin for critical discussions regarding this manuscript and Suellen Greco, DVM DACLAM, for review and selection of pathologic specimens. We would also like to that Stacey Plambeck-Seuss for her technical assistance.

\section{References}

I. Jemal A, Siegel R, Ward E, Hao Y, Xu J, Murray T, et al.: Cancer statistics, 2008. CA Cancer J Clin 2008, 58:7I-96.

2. Metreveli RE, Sahm K, bdel-Misih R, Petrelli NJ: Major pancreatic resections for suspected cancer in a community-based teaching hospital: lessons learned. J Surg Oncol 2007, 95:20I-206.

3. Sierzega M, Popiela T, Kulig J, Nowak K: The ratio of metastaticl resected lymph nodes is an independent prognostic factor in patients with node-positive pancreatic head cancer. Pancreas 2006, 33:240-245.

4. Karasek P, Skacel T, Kocakova I, Bednarik O, Petruzelka L, Melichar B, et al: Gemcitabine monotherapy in patients with locally advanced or metastatic pancreatic cancer: a prospective observational study. Expert Opin Pharmacother 2003, 4:58I-586.

5. Wheeler KT, Wang LM, Wallen CA, Childers SR, Cline JM, Keng PC, et al: Sigma-2 receptors as a biomarker of proliferation in solid tumours. Br J Cancer 2000, 82: 1223-1232.

6. Choi SR, Yang B, Plossl K, Chumpradit S, Wey SP, Acton PD, et al.: Development of a Tc-99m labeled sigma-2 receptor-specific ligand as a potential breast tumor imaging agent. Nucl Med Biol 200I, 28:657-666.

7. Fahy BN, Schlieman MG, Virudachalam S, et al.: Inhibition of AKT abrogates chemotherapy-induced NF-kappaB survival mechanisms: implications for therapy in pancreatic cancer. J Am Coll Surg 2004, 198:59I-599.

8. Flick MB, O'Malley D, Rutherford T, et al.: Apoptosis-based evaluation of chemosensitivity in ovarian cancer patients. J Soc Gynecol Investig 2004, I I:252-259.

9. Martin WR, Eades CG, Thompson JA, Huppler RE, Gilbert PE: The effecs of morphine- and nalorphine- like drugs in the nondependent and morphine- dependent chronic spinal dog. J Pharmachol Exp Ther 1976, 197:517-532.

10. Hou C, Tu Z, Mach R, Kung HF, Kung MP: Characterization of a novel iodinated sigma-2 receptor ligand as a cell proliferation marker. Nucl Med Biol 2006, 33:203-209.

II. Zeng C, Vangveravong S, Xu J, Chang KC, Hotchkiss RS, Wheeler KT, et al.: Subcellular localization of sigma-2 receptors in breast cancer cells using two-photon and confocal microscopy. Cancer Res 2007, 67:6708-6716.

12. Crawford KW, Bowen WD: Sigma-2 receptor agonists activate a novel apoptotic pathway and potentiate antineoplastic drugs in breast tumor cell lines. Cancer Res 2002, 62:313-322.

13. Kashiwagi H, McDunn JE, Simon PO Jr, Goedegebuure PS, Xu J, Jones $L$, et al.: Selective sigma-2 ligands preferentially bind to pancreatic adenocarcinomas: applications in diagnostic imaging and therapy. Mol Cancer 2007, 6:48.

14. Vangveravong S, Xu J, Zeng C, Mach RH: Synthesis of N-substituted 9-azabicyclo[3.3.I]nonan-3alpha-yl carbamate analogs as sigma2 receptor ligands. Bioorg Med Chem 2006, 1 4:6988-6997.

15. Ostenfeld MS, Fehrenbacher N, Hoyer-Hansen M, et al.: Effective tumor cell death by sigma-2 receptor ligand siramesine involves lysosomal leakage and oxidative stress. Cancer Res 2005, 65:8975-8983.

16. Schnell MA, Hardy C, Hawley M, Propert KJ, Wilson JM: Effect of blood collection technique in mice on clinical pathology parameters. Hum Gene Ther 2002, I3(I): I55-6I.

17. Jones $S$, Zhang $X$, Parsons DW, et al.: Core signaling pathways in human pancreatic cancers revealed by global genomic analyses. Science 2008, 32 I(5897): I80I-6.

18. Wagner KW, King F, Nomoto K, et al.: Activation and suppression of the TRAIL death receptor pathway in chemotherapy sensitive and resistant follicular lymphoma cells. Cancer Biol Ther 2003, 2:534-540.

19. Vivo C, Liu W, Broaddus VC: c-Jun $\mathbf{N}$-terminal kinase contributes to apoptotic synergy induced by tumor necrosis factorrelated apoptosis-inducing ligand plus DNA damage in chemoresistant, p53 inactive mesothelioma cells. J Biol Chem 2003, 278:2546I-25467.

20. Boutonnat J, Barbier M, Muirhead K, et al:: Response of chemosensitive and chemoresistant leukemic cell lines to drug therapy: simultaneous assessment of proliferation, apoptosis, and necrosis. Cytometry 2000, 42:50-60.

21. Wang $X$, Wang C, Qin YW, et al:: Simultaneous suppression of multidrug resistance and antiapoptotic cellular defense induces apoptosis in chemoresistant human acute myeloid leukemia cells. Leuk Res 2007, 3 I:989-994.

Publish with Bio Med Central and every scientist can read your work free of charge

"BioMed Central will be the most significant development for disseminating the results of biomedical research in our lifetime. "

Sir Paul Nurse, Cancer Research UK

Your research papers will be:

- available free of charge to the entire biomedical community

- peer reviewed and published immediately upon acceptance

- cited in PubMed and archived on PubMed Central

- yours - you keep the copyright

Submit your manuscript here:

http://www.biomedcentral.com/info/publishing_adv.asp
BioMedcentral 